\title{
Localization and Mobility Gap in Topological Anderson Insulator
}

\author{
Yan-Yang Zhang, Rui-Lin Chu, Fu-Chun Zhang and Shun-Qing Shen ${ }^{1}$ \\ ${ }^{1}$ Department of Physics, The University of Hong Kong, Pokfulam, Hong Kong, China
}

(Dated: November 9, 2018)

\begin{abstract}
It has been proposed that disorder may lead to a new type of topological insulator, called topological Anderson insulator (TAI). Here we examine the physical origin of this phenomenon. We calculate the topological invariants and density of states of disordered model in a super-cell of 2dimensional $\mathrm{HgTe} / \mathrm{CdTe}$ quantum well. The topologically non-trivial phase is triggered by a band touching as the disorder strength increases. The TAI is protected by a mobility gap, in contrast to the band gap in conventional quantum spin Hall systems. The mobility gap in the TAI consists of a cluster of non-trivial subgaps separated by almost flat and localized bands.
\end{abstract}

PACS numbers: 71.23.-k, 73.21.-b, 73.43.Nq,

\section{INTRODUCTION}

Recent development in topological insulators (TI) has greatly enhanced our understanding of topological properties in condensed matter [1 $[3]$. Band insulators with time reversal symmetry can be classified by a $Z_{2}$ topological invariant $\nu$ associated with the occupied bands, $\nu=0$ for topologically trivial phase and $\nu=1$ for non-trivial phase [4 7]. In two dimensions (2D), the TI $(\nu=1)$ exhibits quantum spin Hall effect, whose edge currents are robust against weak non-magnetic disorder [4, 8, 9]. This dissipation-less transport can only be destroyed by extremely strong disorder, which drives the system into a traditional Anderson insulator 10, 11]. The 2D TI has been experimentally realized in $\mathrm{HgTe} / \mathrm{CdTe}$ quantum wells, where the thickness of the quantum well can be varied to tune the system between TI and normal insulator [12, 13.

Recent numerical simulation reveals an interesting new phase, called topological Anderson insulator (TAI) 10]. The TAI is a reentrant TI due to disorder: the disorder drives a 2D topologically trivial insulator into a TI phase, then back to trivial insulator at strong disorder. This is contrary to the general intuition that disorder always tends to localize electronic states. This TAI phase has since attracted extensive research interests 1420 . In the original work, this phase was identified from the transport properties showing a two-terminal conductance plateau $2 e^{2} / h$ with extremely small fluctuations 10$]$. Further numerical studies confirmed that the plateau conductance in the TAI is contributed from the dissipationless edge states 14, which further suggests the topological origin of this phenomena. Theoretical study based on the first Born approximation of the disordered Dirac fermions proposed that the TAI originates from a band touching and subsequent re-opening of a topologically nontrivial gap driven by disorder [15]. The band touching has been confirmed in the perturbative and numerical calculations, but it is not sufficient to explain the whole region in the TAI. Very recently, there has been a phase diagram for the disordered $\mathrm{HgTe} / \mathrm{CdTe}$ quantum spin Hall well, where the quantum spin Hall phase and the TAI are connected 21].

In this paper, we study the topological evolution of the TAI, and examine the origin of the TAI from a topological point of view. We calculate the band structure and the corresponding $Z_{2}$ invariants as the disorder strength increases. Starting with a topologically trivial insulating phase, the bulk gap closes due to the disorder, which changes the topological invariants of the occupied bands, therefore triggering an insulator-TI transition (band inversion). As the disorder strength further increases, a bulk gap is re-opened. However, the gap value is too small (due to large sample size) and too fluctuating (due to the randomness of disorder) to account for a stable TAI phase as observed in transport calculations [10]. We shall show clear evidences that the TAI phase corresponds to a continuous cluster of nontrivial subgaps, rather than a single gap. These subgaps are separated by some extremely narrow subbands, and survive through size scaling and random statistics. In other words, in the TAI region, a Fermi level falls into a nontrivial subgap with a probability close to 1 , regardless of sample size and disorder fluctuations. On the other hand, those extremely narrow subbands are strongly localized, therefore they do not contribute to electronic transport in the thermodynamic limit. This novel phase offers a new realization of quantum spin Hall states in solids.

This paper is organized as follows. In section II, we describe the model we use. In section III, the general definition and calculation methods of topological invariants are reviewed. In section IV, the ansatz of defining topological invariants for disordered systems is introduced. The main results are described in sections V and VI.

\section{THE MODEL}

We first briefly revisit the Bloch's description of electronic properties. In real space, the electronic Hamilto- 
nian in a crystal lattice has the general form

$$
\mathcal{H}=\sum_{i} \sum_{\alpha \beta} \mathcal{H}_{\alpha, \beta}(i, i) c_{i \alpha}^{\dagger} c_{i \beta}+\sum_{\langle i j\rangle} \sum_{\alpha \beta} \mathcal{H}_{\alpha \beta}(i, j) c_{i \alpha}^{\dagger} c_{j \beta},
$$

where $i, j$ are the indices of primary unit cells of the lattice, and $\alpha, \beta$ are the indices of freedom degree within the unit cell, e.g., sublattices, orbitals and spins etc. After Fourier transformation $c_{i \alpha}=\frac{1}{\sqrt{V}} \sum_{\boldsymbol{k}} c_{\boldsymbol{k} \alpha} e^{i \boldsymbol{k} \cdot \boldsymbol{x}_{i}}$, the Hamiltonian can be written as

$$
\mathcal{H}=\sum_{\boldsymbol{k}} \sum_{\alpha \beta} H_{\alpha \beta}(\boldsymbol{k}) c_{\boldsymbol{k} \alpha}^{\dagger} c_{\boldsymbol{k} \beta},
$$

where $\boldsymbol{k}$ is defined in the first Brillouin zone. In the eigenproblem

$$
\sum_{\beta} H_{\alpha \beta}(\boldsymbol{k}) u_{n, \beta}(\boldsymbol{k})=E_{n}(\boldsymbol{k}) u_{n, \alpha}(\boldsymbol{k}),
$$

$E_{n}(\boldsymbol{k})$ determines the band structure, and $\left|u_{n}\right\rangle$ is the unit cell periodic part of the Bloch function $\left|\psi_{n \boldsymbol{k}}\right\rangle=$ $e^{i \boldsymbol{k} \cdot \boldsymbol{r}}\left|u_{n}(\boldsymbol{k})\right\rangle$.

The Bernevig-Hughes-Zhang (BHZ) model[9], a typical tight-binding model with spin-orbit coupling that exhibits quantum spin Hall phase, is defined on a square lattice with one $s$ orbital and one $p$ orbital on each site. In the above mentioned representation, the Bloch Hamiltonian $H$ is a $4 \times 4$ matrix written as

$$
\begin{aligned}
H_{\alpha \beta}(\boldsymbol{k}) & =\left(\begin{array}{cc}
h(\boldsymbol{k}) & g(\boldsymbol{k}) \\
g^{\dagger}(\boldsymbol{k}) & h^{*}(-\boldsymbol{k})
\end{array}\right) \\
h(\boldsymbol{k}) & =d_{0} I_{2 \times 2}+d_{1} \sigma_{x}+d_{2} \sigma_{y}+d_{3} \sigma_{z} \\
g(\boldsymbol{k}) & =\left(\begin{array}{cc}
0 & -\Delta \\
\Delta & 0
\end{array}\right) \\
d_{0}(\boldsymbol{k}) & =-2 D\left(2-\cos k_{x}-\cos k_{y}\right) \\
d_{1}(\boldsymbol{k}) & =A \sin k_{x}, \quad d_{2}(\boldsymbol{k})=-A \sin k_{y} \\
d_{3}(\boldsymbol{k}) & =M-2 B\left(2-\cos k_{x}-\cos k_{y}\right) .
\end{aligned}
$$

Here $\alpha, \beta$ are the indices of spinorbital within the unit cell, $\alpha, \beta \in\{1,2,3,4\} \equiv\{|s \uparrow\rangle,|p \uparrow\rangle,|s \downarrow\rangle,|p \downarrow\rangle\} . \sigma_{i}$ are Pauli matrices acting on the spinor space spanned by $s$ and $p$ orbitals. The real space Hamiltonian $\mathcal{H}$ of this model can be obtained from $H_{\alpha \beta}$ by a straightforward inverse Fourier transformation $c_{\boldsymbol{k} \alpha}=\frac{1}{\sqrt{V}} \sum_{i} c_{i \alpha} e^{-i \boldsymbol{k} \cdot \boldsymbol{x}_{i}}$. The effect of non-magnetic impurities is included in real space by adding a term

$$
V_{I}=\sum_{i} \sum_{\alpha} U(i) c_{i \alpha}^{\dagger} c_{i \alpha}
$$

to $\mathcal{H}$, where $U(i)$ are random numbers uniformly distributed in $(-W / 2, W / 2)$.

\section{III. $Z_{2}$ INVARIANT}

For a time reversal invariant system including both spin components, Kramers Theorem states that, all

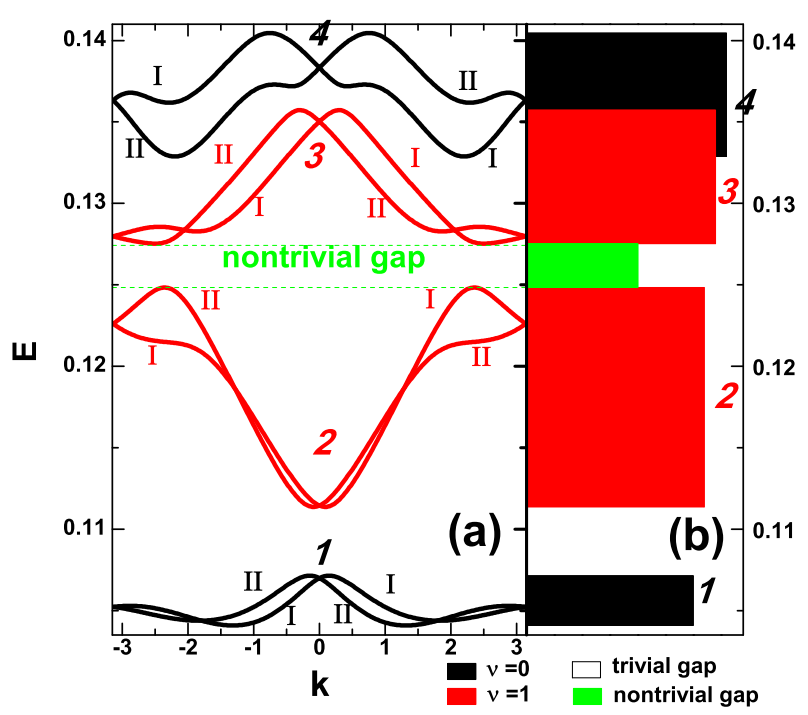

FIG. 1: (Color online) Schematic illustration of Kramers pairs. Black is for trivial pair and red for non-trivial pair. (a) Band structures $E(\boldsymbol{k})$. (b) Extension of the Kramers pair in (a), represented by the width of a solid bar along the energy axis. Different heights (in horizontal direction) of the bars are used to distinguish individual pairs. Green bar is for nontrivial gap.

the electronic bands $E_{n}(\boldsymbol{k})$ come in pairs connected at time reversal invariant points (TRIPs), which are called Kramers pairs [5, 22]. If there are no other degeneracies (e.g., disordered "supercells" which will be discussed in the following) therefore each Kramers pair (KP) is separated from others, a topological invariant can thus be defined for each KP 23]. In Fig. 1] (a), we illustrate the typical band structures of a time reversal invariant system in the topological aspect. There are 8 bands, forming $4 \mathrm{KPs}$, two of which (pairs 2 and 3 in red) are topologically nontrivial, which will be defined below. Pairs 3 and 4 are separated but overlapping in the energy axis. We will simply call the gap between them as not fully gapped. Most of the information in Fig. 1 (a) can be plotted in a simple "bar code" version in 1] (b), where the extensions of the KPs and full gaps along the energy axis are represented by the width of the bars in this direction.

In $2 \mathrm{D}$ the topological invariant $\nu$ associated with a $\mathrm{KP}$ is a $Z_{2}$ integer defined from the periodic part of the Bloch 


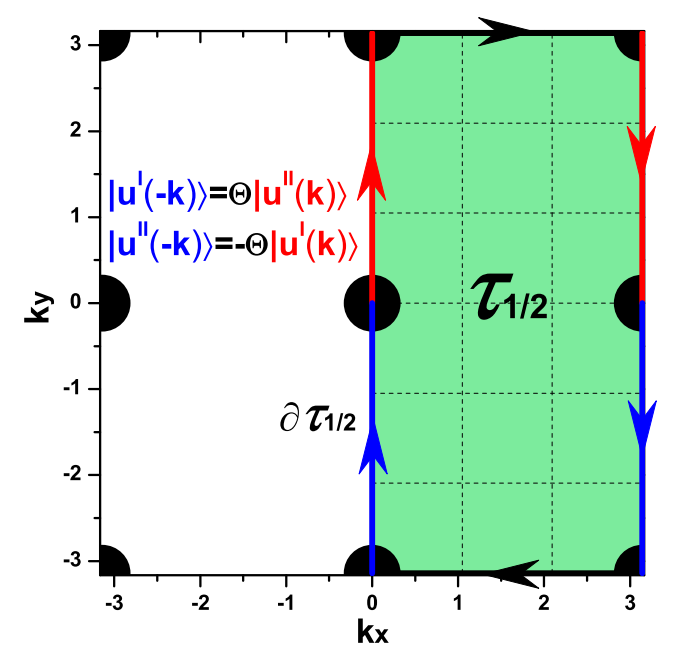

FIG. 2: (Color online) First Brillouin zone of a time reverse symmetric solid in square lattice. Black dots are time reversal invariant points. Green region is the effective Brillouin zone $\tau_{1 / 2}$, and arrows indicate its boundary $\partial \tau_{1 / 2}$. Dashed lines: mesh used in our calculations (8).

function $u(\boldsymbol{k})$ as 22 ]

$$
\begin{aligned}
\nu & =\frac{1}{2 \pi}\left[\oint_{\partial \tau_{1 / 2}} d \boldsymbol{k} \cdot \boldsymbol{A}-\int_{\tau_{1 / 2}} d \boldsymbol{k}^{2} F\right] \bmod 2, \\
\boldsymbol{A}(\boldsymbol{k}) & =\sum_{s=\mathrm{I}, \mathrm{II}} i\left\langle u^{s}(\boldsymbol{k})\left|\nabla_{k}\right| u^{s}(\boldsymbol{k})\right\rangle, \\
F(\boldsymbol{k}) & =\left(\nabla_{\boldsymbol{k}} \times \boldsymbol{A}(\boldsymbol{k})\right)_{z} .
\end{aligned}
$$

Here $\tau_{1 / 2}$ is the effective Brillouin zone (EBZ) from which the rest half can be obtained from its time reverse, $\partial \tau_{1 / 2}$ is the boundary of $\tau_{1 / 2}$, as illustrated in Fig. 2, The Romantic numbers I and II in equation (9) label the two branches of a KP (See Fig. 1 (a)). Note the time reversal constraint

$$
\begin{aligned}
\left|u^{\mathrm{I}}(-\boldsymbol{k})\right\rangle & =\Theta\left|u^{\mathrm{II}}(\boldsymbol{k})\right\rangle \\
\left|u^{\mathrm{II}}(-\boldsymbol{k})\right\rangle & =-\Theta\left|u^{\mathrm{I}}(\boldsymbol{k})\right\rangle
\end{aligned}
$$

on $\partial \tau_{1 / 2}$ must be employed for equation (8) to make sense, where $\Theta=-i s_{y} \otimes I_{2 \times 2} K$ is the time reversal operator $\left(s_{y}\right.$ is the Pauli matrix of physical spin and $K$ here is the complex conjugation). A KP is trivial (nontrivial) if $\nu=0(\nu=1)$. The topological invariant of a cluster of occupied KPs is just the sum of $\nu$ of all these KPs in the sense of mod 2. Therefore, a gap between two pairs are called trivial (nontrivial) if there are even (odd) number of nontrivial pairs below it. If a gap is nontrivial, dissipationless edge states will appear within the gap, when the system is truncated with open boundaries [5, 22, 24].

Among several equivalent definitions of $Z_{2}$ invariant $\nu[5,22,25]$, this definition has the advantages of being expressed by well known topological quantities, i.e., Berry connection $\boldsymbol{A}$ and Berry curvature $\boldsymbol{F}[26$, and being appropriate for numerical evaluation [23, 27, 28], which is briefly introduced here. After discretizing the EBZ into a mesh (dashed lines in Fig.2 (c)), the field quantities $\boldsymbol{A}$ and $\boldsymbol{F}$ can be defined from the eigenstates of the lattice sites [23, 27, 28], based on well-developed lattice gauge theories. Note in the numerical calculations, for any mesh site $\boldsymbol{k}$ in the EBZ, the phases of the eigenstates (therefore the values of the field quantities) are arbitrarily and independently determined by the numerical routines $(U(1)$ freedom of local gauge choice). Care must be taken to cancel all these phase uncertainties when summing up the discretized field quantities $\boldsymbol{A}$ and $\boldsymbol{F}$ by equation (8), so that the resultant $\nu$ is gauge independent. Of course, the mesh should be dense enough to obtain converged values for each KP.

For the clean systems, the topological properties of this model are well understood [5], when the lower half bands are occupied. When $\Delta=0$, the quantum spin Hall phase with $Z_{2}=1$ is realized when $0<M /(2 B)<2$. When tuning $M / B$, a "band inversion" [9, 13] occurs at $\Gamma$ point, leading to a I-TI transition. The presence of $g(\boldsymbol{k})$ breaks the conservation of $S_{z}$, but the topological invariants does not change as long as the finite gap remains.

\section{ZONE FOLDING}

The topological invariants are defined in $\boldsymbol{k}$ space 22, 29], as introduced above. Impurities break the translation invariance of the original lattice and make $\boldsymbol{k}$ badly defined. However, for a disordered 2D sample with $N \times N$ unit cells, the above topological arguments can be restored if twisted boundary conditions

$\psi\left(\boldsymbol{r}+N \cdot \boldsymbol{a}_{1}\right)=e^{i \boldsymbol{k} \cdot N \boldsymbol{a}_{1}} \psi(\boldsymbol{r}), \quad \psi\left(\boldsymbol{r}+N \cdot \boldsymbol{a}_{2}\right)=e^{i \boldsymbol{k} \cdot N \boldsymbol{a}_{2}} \psi(\boldsymbol{r})$

are introduced to the opposite boundaries of this finite sample, where $\boldsymbol{a}_{i}$ are primitive vectors of the clean lattice 23, 30, 31. Physically speaking, this is completely equivalent to taking this $N \times N$ sample as a large unit cell of a 2D super-lattice, so that $\boldsymbol{k}$ can be defined in a smaller Brillouin zone with reciprocal vectors $\boldsymbol{b}_{i} / N$, where $\boldsymbol{b}_{i}$ is the reciprocal vector of the original lattice. Disorder within this supercell tends to destroy all the band degeneracies except those protected by time reversal symmetry, i.e., Kramers degeneracies. It is reasonable to imagine that for sufficiently large $N$, the topological properties of this superlattice can reflect those of the "real" disordered system. In the following, we will call the primary unit cell of the original clean system as a "unit cell", while the $N \times N$ sample as a "supercell" in this context.

In the clean limit, the band structure of the superlattice can be derived directly from that of the original lattice by using the standard method of "zone folding", 
which is briefly reviewed here. Now the Bloch Hamiltonian $H(\boldsymbol{k})$ becomes a $4 N^{2} \times 4 N^{2}$ matrix

$$
H_{i \alpha, j \beta}^{\mathrm{S}}(\boldsymbol{k}), \quad 1 \leq i, j \leq N^{2},
$$

where $\alpha, \beta$ again represent the spinorbital indices within the original unit cell, and $i, j$ are the indices of unit cells within the supercell. The eigenvalues of $H^{\mathrm{S}}(\boldsymbol{k})$ are related with those of the original lattice $E_{n}(\boldsymbol{k})$ as

$$
E_{n, l m}^{\mathrm{S}}(\boldsymbol{k})=E_{n}\left(\boldsymbol{k}+\frac{l}{N} \boldsymbol{b}_{1}+\frac{m}{N} \boldsymbol{b}_{2}\right), \quad 0 \leq l, m \leq N-1
$$

and associated eigenstates are

$$
u_{n, l m}^{\mathrm{S}}(\boldsymbol{k})=\left(\begin{array}{c}
e^{i\left(\frac{l}{N} \boldsymbol{b}_{1}+\frac{m}{N} \boldsymbol{b}_{2}\right) \cdot \boldsymbol{r}_{\mathbf{1}}} u_{n}\left(\boldsymbol{k}+\frac{l}{N} \boldsymbol{b}_{1}+\frac{m}{N} \boldsymbol{b}_{2}\right) \\
e^{i\left(\frac{l}{N} \boldsymbol{b}_{1}+\frac{m}{N} \boldsymbol{b}_{2}\right) \cdot \boldsymbol{r}_{2}} u_{n}\left(\boldsymbol{k}+\frac{l}{N} \boldsymbol{b}_{1}+\frac{m}{N} \boldsymbol{b}_{2}\right) \\
\vdots \\
e^{i\left(\frac{l}{N} \boldsymbol{b}_{1}+\frac{m}{N} \boldsymbol{b}_{2}\right) \cdot \boldsymbol{r}_{N} \times N} u_{n}\left(\boldsymbol{k}+\frac{l}{N} \boldsymbol{b}_{1}+\frac{m}{N} \boldsymbol{b}_{2}\right)
\end{array}\right) .
$$

Equations (14) and (15) can be verified by a straightforward application of Bloch's Theorem, with the new definitions of supercell and associated Brillouin zone in mind.

\section{A SMALL SUPERCELL}

We will only consider the BHZ model in the case of $|D|<|B|$, so that the system is always fully gaped between the lower and upper halves of bands, when $M \neq 0$. To obtain some insights from analytical treatments, we start from a simple stage, a small supercell with $2 \times 2$ unit cells without spin-flip parts, i.e., $\Delta=0$. Now the system is decoupled into two sub-systems with single spin component, and the topological property of $H_{4 \times 4}(\boldsymbol{k})$ in equation (4) can be reduced to that of $h_{2 \times 2}(\boldsymbol{k})$, represented by spin-resolved Chern number [31, 32]. To further simplify the analytical treatments, we can only consider the spin-up sub-system, because its spin-down counterpart can be obtained from a straightforward time reversal operation. For this spin-up sub-system with a $2 \times 2$-site supercell, the Hamiltonian is a $8 \times 8$ matrix

$$
h_{I}^{\mathrm{S}}=h^{\mathrm{S}}(\boldsymbol{k})+V_{I}^{\mathrm{S}}
$$

where $h_{8 \times 8}^{\mathrm{S}}(\boldsymbol{k})$ is constructed from the above zone-folding technique from the original $h_{2 \times 2}(\boldsymbol{k})$ in equation (5) and the impurity term reads

$$
V_{I}=W \cdot \operatorname{diag}\left(\epsilon_{1}, \epsilon_{1}, \epsilon_{2}, \epsilon_{2}, \epsilon_{3}, \epsilon_{3}, \epsilon_{4}, \epsilon_{4}\right),
$$

where $\epsilon_{i}$ are random numbers within the interval $(1 / 2,1 / 2)$ and $W$ is a single parameter to control the disorder strength. This $V_{I}$ represents random onsite potential distributed on 4 primary unit cells within the $2 \times 2$ super-cell. We will show that, this minimal model in equation (16) that accommodates both disorder and topology, can produce some non-trivial results.

Without impurities, as stated above, the eigenenergies and eigenstates of $h^{\mathrm{S}}$ can be constructed from those of $h$ by zone-folding, eqs. (14) and (15). The eigenenergies of $h^{\mathrm{S}}$ are ordered by their values at the $\Gamma$ point $\boldsymbol{k}=0$ as

$$
\begin{aligned}
& E_{1,2, \cdots, 8}^{0}(\Gamma)=-8 D-|M-8 B|, \quad-4 D-|M-4 B|, \\
& -4 D-|M-4 B|, \quad-M, \quad M, \quad-4 D+|M-4 B|, \\
& -4 D+|M-4 B|, \quad-8 D+|M-8 B|,
\end{aligned}
$$

with a gap $2 M$ between conductance band $E_{4}$ and valance band $E_{5}$. The presence of $V_{I}$ will change band structures. Although the band structures including impurities can be deduced from diagonalizing eq. (16) directly, we will treat the impurities as a perturbation, therefore the eigenenergies can be expressed as matrix elements between unperturbed eigenstates $\left|u_{i}^{\mathrm{S}}\right\rangle$. Straightforward calculations show that at $\Gamma=(0,0)$, the first order correction to these two states is

$$
\begin{aligned}
& E_{4}^{(1)}(\Gamma)=\left\langle u_{4}^{S}(\Gamma)\left|V_{I}\right| u_{4}^{S}(\Gamma)\right\rangle=\frac{W}{4}\left(\epsilon_{1}+\epsilon_{2}+\epsilon_{3}+\epsilon_{4}\right) \\
& E_{5}^{(1)}(\Gamma)=\left\langle u_{5}^{S}(\Gamma)\left|V_{I}\right| u_{5}^{S}(\Gamma)\right\rangle=\frac{W}{4}\left(\epsilon_{1}+\epsilon_{2}+\epsilon_{3}+\epsilon_{4}\right),
\end{aligned}
$$

which is just a uniform shift as a simple mean field of impurity potentials. The second order correction is

$$
\begin{aligned}
& E_{4}^{(2)}(\Gamma)=\sum_{i \neq 4} \frac{\left|\left\langle u_{4}^{S}(\Gamma)\left|V_{I}\right| u_{i}^{S}(\Gamma)\right\rangle\right|^{2}}{E_{4}(\Gamma)-E_{i}(\Gamma)}=\frac{-W^{2} F\left(\epsilon_{1}, \epsilon_{2}, \epsilon_{3}, \epsilon_{4}\right)}{128(B-D)} \\
& E_{5}^{(2)}(\Gamma)=\sum_{i \neq 5} \frac{\left|\left\langle u_{5}^{S}(\Gamma)\left|V_{I}\right| u_{i}^{S}(\Gamma)\right\rangle\right|^{2}}{E_{5}(\Gamma)-E_{i}(\Gamma)}=\frac{W^{2} F\left(\epsilon_{1}, \epsilon_{2}, \epsilon_{3}, \epsilon_{4}\right)}{128(B+D)},
\end{aligned}
$$

where

$$
\begin{aligned}
& F\left(\epsilon_{i}\right)=5\left(\epsilon_{1}^{2}+\epsilon_{2}^{2}+\epsilon_{3}^{2}+\epsilon_{4}^{2}\right)-2\left(\epsilon_{1} \epsilon_{2}+\epsilon_{2} \epsilon_{3}+\epsilon_{1} \epsilon_{4}+\epsilon_{3} \epsilon_{4}\right) \\
& -6\left(\epsilon_{1} \epsilon_{3}+\epsilon_{2} \epsilon_{4}\right) \begin{cases}=0, & \epsilon_{1}=\epsilon_{2}=\epsilon_{3}=\epsilon_{4} \\
>0, & \text { otherwise }\end{cases}
\end{aligned}
$$

is a semi-positive-definite quadrics. Now the gap is

$$
\begin{aligned}
E_{g} & =\left|\left(E_{5}^{0}+E_{5}^{(1)}+E_{5}^{(2)}\right)-\left(E_{4}^{0}+E_{4}^{(1)}+E_{4}^{(2)}\right)\right| \\
& =\left|2 M\left(1+\frac{B}{M} \cdot \frac{W^{2} F\left(\epsilon_{1}, \epsilon_{2}, \epsilon_{3}, \epsilon_{4}\right)}{64\left(B^{2}-D^{2}\right)}\right)\right|
\end{aligned}
$$

Equation (18) is the first important result in this paper. It suggests that in the weak disorder regime, the impurities effectively renormalize $M[15]$ and this renormalization comes from a second order effect of disorder. Note the sign of this renormalization term $\frac{B}{M} \cdot \frac{W^{2} F\left(\epsilon_{i}\right)}{64\left(B^{2}-D^{2}\right)}$ does not depend on the concrete configuration of random impurities. If the clean system is topologically non-trivial $(M / B>0)$, the gap grows as $M+$ const. $W^{2}$. This means that weak disorder tends to make the two bands expel 


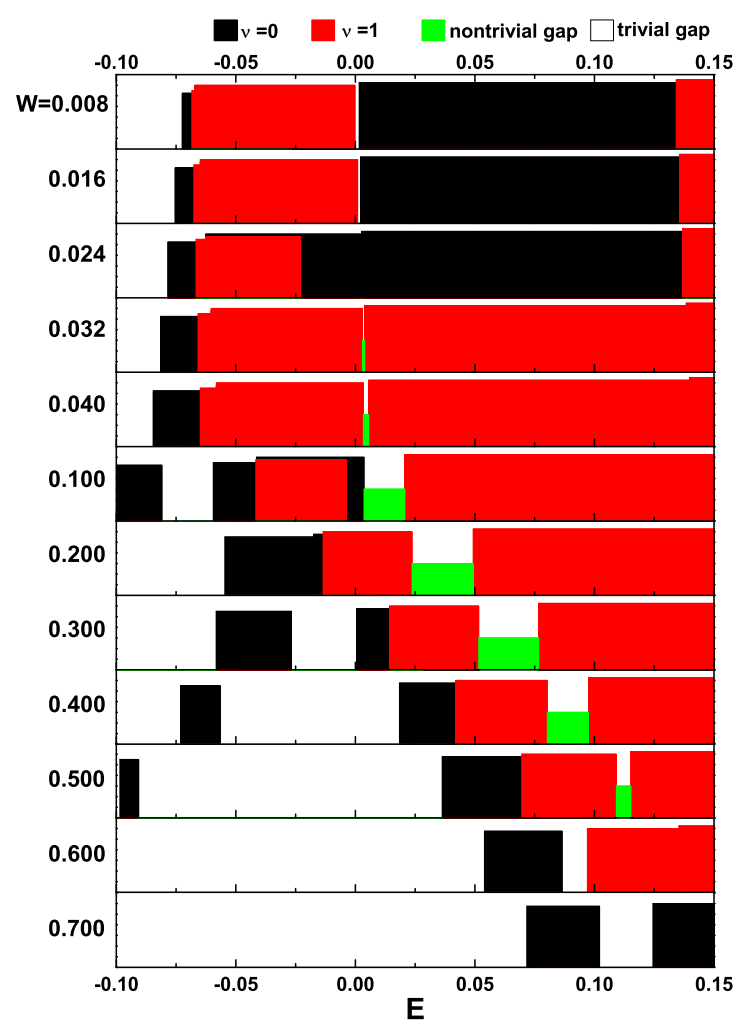

FIG. 3: Evolution of Kramers pairs and their topological invariants for a $2 \times 2$ supercell as disorder strength $W$ increase (top to bottom), for a definite configuration of $\left\{\epsilon_{i}\right\}$. Bars: same as in Fig. 1(b). Model parameters for $\mathrm{H}$ in Eqs. (4): $A=0.0729, B=-0.0274, C=0, D=-0.205, M=0.001$ and $\Delta=0$, which are also consistent with [10, 14]. Lattice constant is set to be 1 .

each other to avoid a band-touching which will trigger a transition to a trivial insulator [11]. This is a vivid manifestation of "robustness against weak disorder" for TI. If the clean system is topologically trivial $(M / B<0)$, on the other hand, the gap decays as $M-$ const $\cdot W^{2}$. If $M$ is small, when we tune disorder on, the gap will soon close at some small $W_{c}$, before strong disorder makes the above perturbation treatment unreliable. This gap close leads to an I-TI transition with the sign change of $M$. From the topological point of view, the Chern number of $E_{4}$ changes by 1 after band-touching 11,33 , 34]. Remember we only considered the spin-up block so far, but the physical argument of topologically trivial-nontrivial transition also applies when the time-reversal invariant $H$ involving both spin blocks is considered, by a simple correspondence between Chern number and $Z_{2}$ invariant [6], as long as these two blocks are decoupled.

To test the above physical pictures, we calculate the $Z_{2}$ topological invariants $\nu$ for a $2 \times 2$ supercell with both spin components included. In Fig. 3 the evolution of KPs for a definite configuration of $\left\{\epsilon_{i}\right\}$ with increasing disorder strength $W$ are plotted. We can see that the gap closing predicted by second order perturbation really happens at $W=0.024$ and it does lead to a topological transition from $\nu=0$ to $\nu=1$ associated with the lower half bands. After this transition, a topologically non-trivial gap (the green bar) emerges. This gap will develop with further increasing $W$, until strong disorder eventually close it again 11]. This disorder-induced nontrivial gap shifts towards positive energy with increasing $W$, as the TAI region observed in [10] does. This simple model itself also paves a route to producing a TI phase from a trivial insulator with spin-orbit coupling by constructing a superlattice 35 .

\section{LARGE SUPERCELLS}

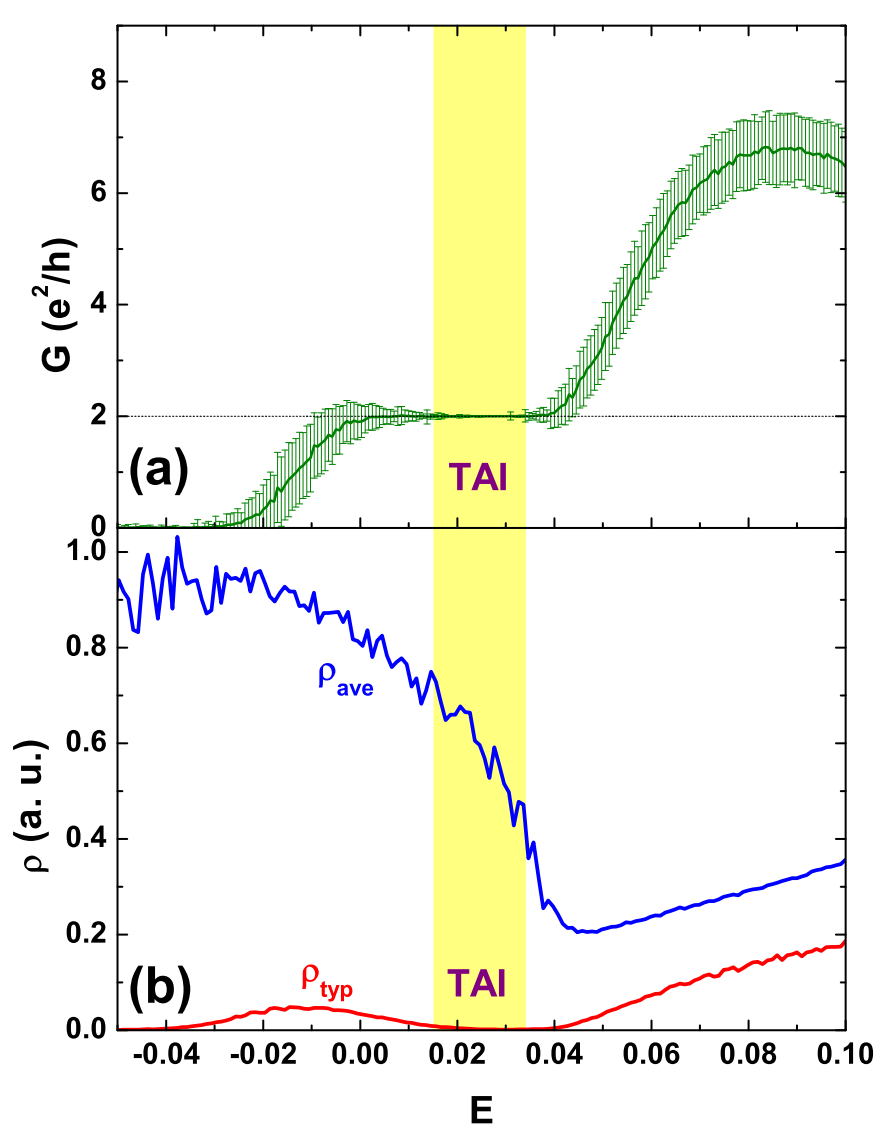

FIG. 4: (a) Two-terminal conductance of a samples with size $100 \times 100$, as functions of energy at a given disorder strength $W=0.2$. Conductance is an average over 300 random configurations. Conductance plateau with extremely small fluctuations corresponds to the TAI phase, indicated by the light yellow region. (b) The average $\operatorname{DOS} \rho_{\text {ave }}$ (blue) and the typical DOS $\rho_{\text {typ }}$ (red), calculated from 300 samples with size $100 \times 100$ and with periodic boundary conditions. The parameters are the same as in Fig. 3

So far, the origin of TAI seems clear: the disorder triggers a band touching, or a band inversion, after which 
a nontrivial gap opens for the TAI phase to live. Unfortunately, this simple argument from a small supercell cannot directly be applied to large samples, which will be

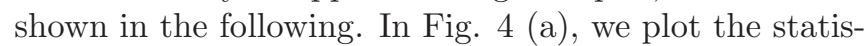
tics of two-terminal conductance of a $100 \times 100$ samples. The two-terminal conductance is calculated by the standard non-equilibrium Green's function method 36], and the Fermi energy in the leads are fixed at $E_{F}^{\text {lead }}=0.12$ to offer enough number of channels. The TAI phase is identified as the conductance plateau $2 e^{2} / h$ with extremely small fluctuations, as in Ref. [10]. If this region corresponds to a bulk gap, the density of states (DOS) must vanish, at least in the case of periodic boundary condition. The single particle local density of states (LDOS) is calculated as 37.

$$
\rho(i, E)=\frac{1}{N^{2}} \sum_{n}|\langle i \mid n\rangle|^{2} \delta\left(E-E_{n}\right) .
$$

The arithmetic mean of the LDOS

$$
\rho_{\text {ave }}(E) \equiv \ll \rho(i, E) \gg
$$

is just the bulk DOS except for a constant factor, where $\ll \cdots \gg$ is the arithmetic average over the sites of the sample. Meanwhile, the geometric mean of the LDOS

$$
\rho_{\text {typ }}(E) \equiv \exp [\ll \ln \rho(i, E) \gg]
$$

gives the localization property of the states. In the thermodynamic limit $(N \rightarrow \infty)$, if $\rho_{\text {ave }}(E) / \rho_{\text {typ }}(E) \rightarrow 0$, then the states around $E$ is localized [38]. We thus plot $\rho_{\text {ave }}$ and $\rho_{\text {typ }}$ in Fig. 4 (b) in the case of periodic boundary conditions. Two remarkable features can be read

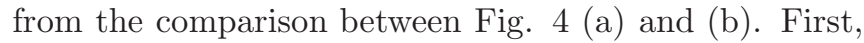
the DOS $\rho_{\text {ave }}$ does not vanish in TAI region. As a matter of fact, there are regions with smaller DOS outside the TAI region. In other words, the TAI phase does not live in a bulk gap at all. Second, the TAI region corresponds to a vanishing of $\rho_{\text {typ }}$. This means that these states are extremely localized.

These surprising results throw a doubt on whether TAI can be understood within the above mentioned topological regime. In order to answer this, we repeat the numerical calculation of $\nu$ for larger supercells. In Fig. [5 we plot the evolution of KPs for a $8 \times 8$ supercell associated with a definite configuration of $\left\{\epsilon_{i}\right\}$, with increasing disorder strength $W$. There is also a band touching at $W=0.05$, which triggers a nontrivial subgap represented by a green bar, as in the case of a small supercell. On the other hand, with stronger disorder, for example, around $W=0.2$, it develops into a wider region of nontrivial subgaps separated by narrow KPs, instead of one single nontrivial gap. In Fig. [6 we show the average width of KPs within the TAI region, it is clear that in the thermodynamic limit, the KPs will be extremely narrow. These narrow KPs are topologically trivial 39 41] and do not affect the topology (trivial or nontrivial)

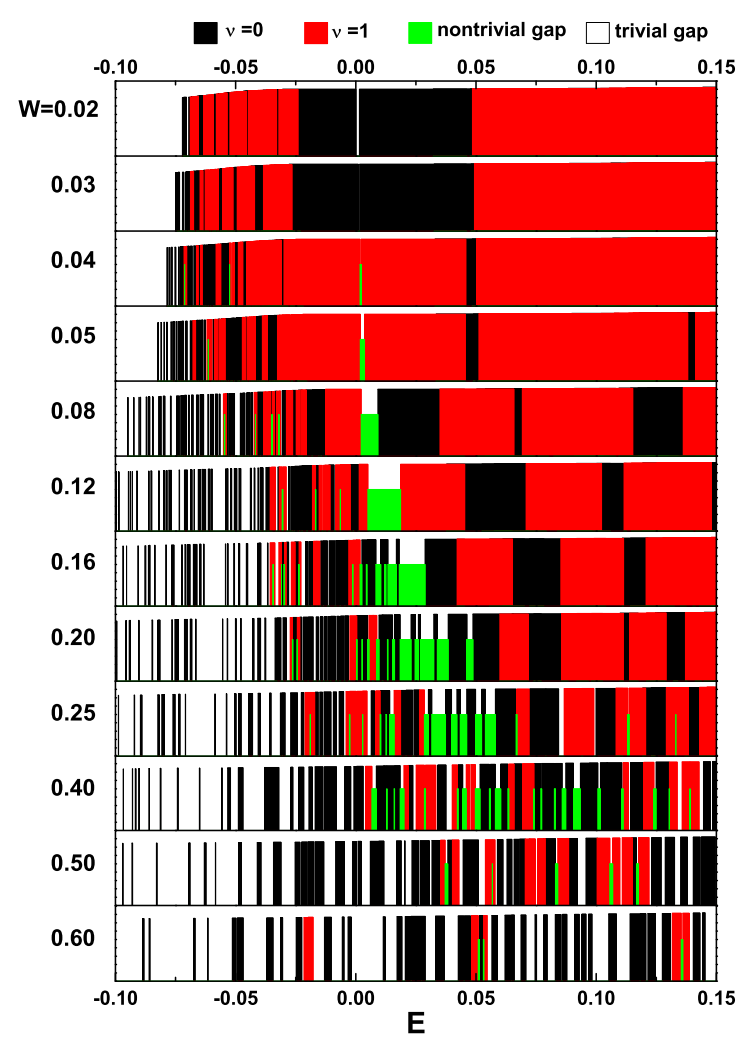

FIG. 5: Evolution of Kramers pairs and their topological invariants for a $8 \times 8$ supercell, for a definite configuration of $\left\{\epsilon_{i}\right\}$. Disorder strength $W$ increases from top to bottom. Parameters are the same as in Fig. 3

of subgaps between them. In other words, the disorder induced nontrivial nature soon hides in the lower KPs deeply below the TAI region. We will argue that, those narrow and topologically trivial KPs are responsible for nonzero DOS in this region, while these nontrivial subgaps are responsible for the TAI region observed from transport calculations in Ref. [10].

The nonzero DOS is easy to understand. Although the KPs are microscopically separated, but due to the broadening $\eta$ associated with any measure or calculation of DOS, they will give rise to a continuous region of finite DOS. Moreover, these flat and well separated KPs tend to be strong localized [23]. This is what we have observed in Fig. 4 (b). One may also imagine that a fluctuating transition disorder strength $W_{c}$ from sample to sample might contribute to the statistically nonzero DOS in the TAI region. However, as stated in section V, since this gap closing is a perturbation effect at weak disorder, the fluctuation of $W_{c}$ is also very small. Indeed, the numerical results confirm that (not shown here), compared to the width of TAI region, the statistical error of $W_{c}$ is extremely small.

On the other hand, the origin of TAI phase exhibited from transport calculations is more profound. It is well 


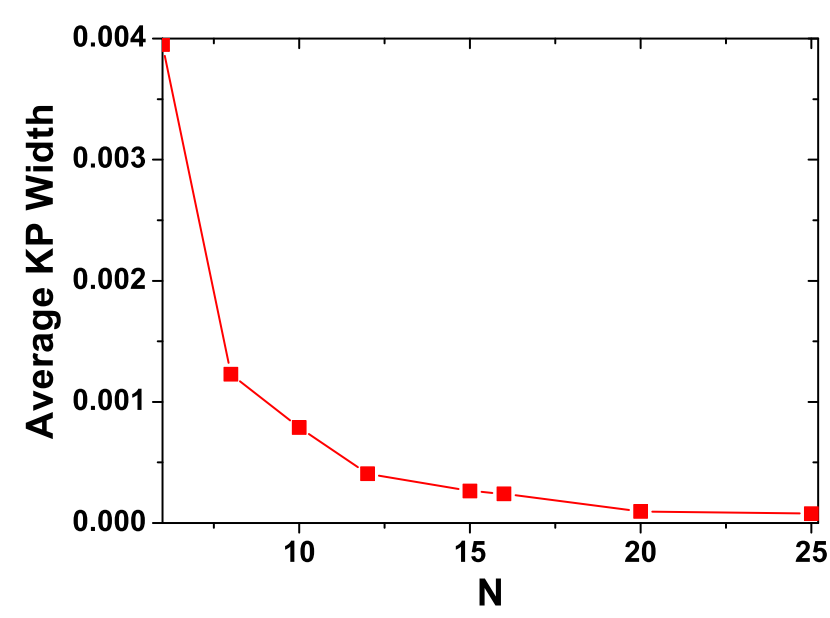

FIG. 6: (Color online) Average width of Kramers pairs between $E=0.01$ and $E=0.03$ as a function of supercell size $N$. Parameters are the same as in Fig. 3

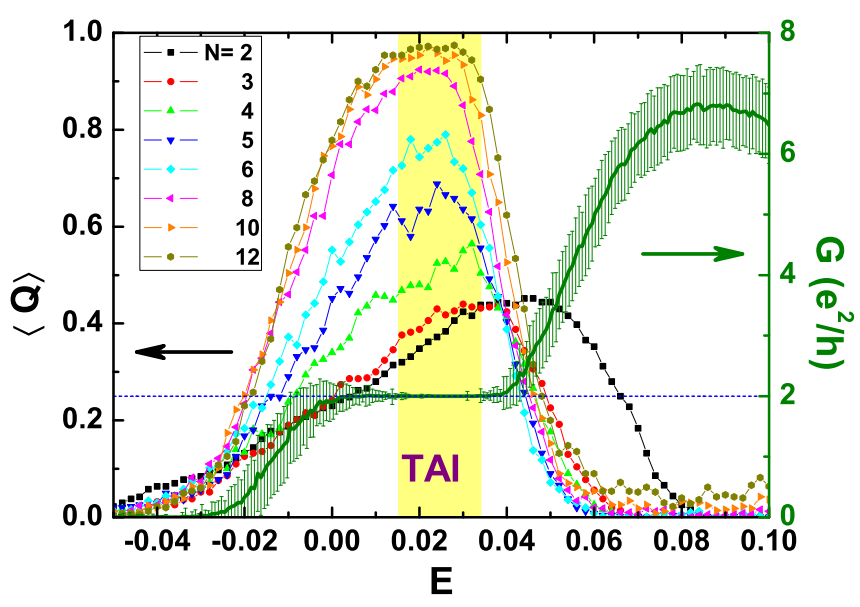

FIG. 7: (Color online) Probability of falling into a nontrivial subgap $\langle Q\rangle$ (lines with symbols) for supercells with different size $N$, at $W=0.2$. Every $\langle Q\rangle$ is averaged over 500 random configurations. Green curve is the conductance, same as that in Fig. 4 Parameters are the same as in Fig. 3

known that a nontrivial subgap always gives rise to dissipationless edge states. However, in our case, these disorder induced nontrivial subgaps are densely and randomly distributed on the energy axis. To confirm that they are indeed responsible for TAI phase, one must verify that these nontrivial subgaps can survive through random statistics and size scaling. To characterize this quantitatively, we define a function

$$
Q(E)=\left\{\begin{array}{l}
1, E \in \text { a nontrivial and full subgap } \\
0, \text { otherwise }
\end{array}\right.
$$

for a definite supercell size and a definite disorder configuration. The average $\langle Q(E)\rangle$ over the disorder ensemble is the probability for the Fermi energy $E$ to fall into a nontrivial subgap. If the above physical picture can make sense, $\langle Q(E)\rangle$ must be a value very close to 1 in the TAI region, under random averaging and size scaling. In Fig. 7. we show the numerical results of $\langle Q(E)\rangle$ over averaging and scaling. The conductance curve is also plotted as a comparison. It is happy to see that, the $\langle Q(E)\rangle$ curves converge to a broad peak approaching 1 , and that the energy region of the broad peak does correspond to the conductance plateau of TAI. Note this broad peak of $\langle Q(E)\rangle$ calculated with supercell size $\geq 10 \times 10$ is sufficient to reproduce TAI region identified from conductance plateau for $100 \times 100$ samples. In the process of scaling $(N \rightarrow \infty)$, the numbers of subpairs and subgaps increase, while the widths of individual subpairs and subgaps decrease but with different decreasing rate. As a result, as disclosed in Fig. 77 for large enough $N$, the total measure of subgaps will dominate over that of flat subpairs, $\langle Q\rangle \sim 1 \gg 1-\langle Q\rangle$. Fig. 7 is the most important result of this paper. It reveals that, the TAI phase corresponds to a cluster of nontrivial subgaps instead of a single topologically nontrivial gap. A Fermi energy falls into a nontrivial subgap with a probability close to 1 . The KPs, although contributing to nonzero DOS in this region, are so narrow that their measures on the energy axis are extremely small, and they are localized therefore do not contribute to the electronic transport. Because of the topological origin, it is now clear why the TAI is an QSHE phase seen from the real space 21], and why the dissipationless currents are still carried by edge states [14].

\section{SUMMARY}

In summary, the topological evolution of TAI is studied in a supercell regime. Starting from a trivial insulator phase with a small gap, weak disorder inevitably lead to gap closing between the valence and conduction bands, which is a second order perturbation effect. This causes an exchange of topological invariants between them, and results in a transition to a topologically nontrivial phase. In the limit of large supercell, there will be very large numbers of subbands and subgaps densely distributed on the energy axis. However, there exists a continuous region where the Fermi energy falls into a nontrivial subgap with an extremely high probability, even after a statistical average over the disorder ensemble. This special region can thus support a stable and observable TAI phase. This physical picture also helps find disorder-induced topological insulators in other materials and higher dimensions [20].

\section{ACKNOWLEDGEMENTS}

This work was supported by the Resarch Grant Council of Hong Kong under Grant No. HKU705110P. 
[1] M. Z. Hasan and C. L. Kane, Rev. Mod. Phys. 82, 3045 (2010).

[2] X. L. Qi and S. C. Zhang, Phys. Today 63, 33 (2010)

[3] J. E. Moore, Nature 464, 194 (2010)

[4] C. L. Kane and E. J. Mele, Phys. Rev. Lett. 95, 146802 (2005).

[5] L. Fu and C. L. Kane, Phys. Rev. B 76, 045302 (2007).

[6] J. E. Moore and L. Balents, Phys. Rev. B 75, 121306(R) (2007).

[7] X.-L. Qi, T. L. Hughes and S.-C. Zhang, Phys. Rev. B 78, 195424 (2008).

[8] C. L. Kane and E. J. Mele, Phys. Rev. Lett. 95, 226801 (2005).

[9] A. Bernevig, T. Hughes and S. C. Zhang, Science 314, 1757 (2006).

[10] J. Li, R.-L. Chu, J. K. Jain and S.-Q. Shen, Phys. Rev. Lett. 102, 136806 (2009).

[11] Y. Hatsugai, K. Ishibashi and Y. Morita, Phys. Rev. Lett. 83, 2246 (1999).

[12] M. Konig, S. Wiedmann, C. Brune, A. Roth, H. Buhmann, L. W. Molenkamp, X. L. Qi and S. C. Zhang, Science 318, 766 (2007).

[13] M. König, H. Buhmann, L. W. Molenkamp, T. Hughes, C.-X. Liu, X.-L. Qi and S.-C. Zhang, J. Phys. Soc. Jpn. 77, 031007 (2008).

[14] H. Jiang, L. Wang, Q.-F. Sun and X. C. Xie, Phys. Rev. B 80, 165316 (2009).

[15] C. W. Groth, M. Wimmer, A. R. Akhmerov, J. Tworzydło and C. W. J. Beenakker, Phys. Rev. Lett. 103, 196805 (2009).

[16] A. Yamakage, K. Nomura, K. Imura, Y. Kuramoto, J. Phys. Soc. Jpn. 80053703 (2011)

[17] L. Chen, Q. Liu, X. Lin, X. G. Zhang, and X. Y. Jiang, arXiv: 1106.4103

[18] Y. X. Xing, L. Zhang, and J. Wang, Phys. Rev. B 84, $035110(2011)$

[19] W. Li, J. D. Zang, and Y. J. Jiang, Phys. Rev. B 84, 033409 (2011)

[20] H.-M. Guo, G. Rosenberg, G. Refael and M. Franz, Phys.
Rev. Lett. 105, 216601 (2010).

[21] E. Prodan, Phys. Rev. B 83, 195119 (2011).

[22] L. Fu and C. L. Kane, Phys. Rev. B 74, 195312 (2006).

[23] A. M. Essin and J. E. Moore, Phys. Rev. B 76, 165307 (2007).

[24] L. Fu, C. L. Kane and E. J. Mele, Phys. Rev. Lett. 98, 106803 (2007).

[25] Z. Wang, X.-L. Qi and S.-C. Zhang, New J. Phys. 12, 065007 (2010).

[26] D. Xiao, M.-C. Chang and Q. Niu, Rev. Mod. Phys. 82, 1959 (2010).

[27] T. Fukui and Y. Hatsugai, J. Phys. Soc. Jpn. 76, 053702 (2007).

[28] D. Xiao, Y. G. Yao, W. X. Feng, J, Wen, W. G. Zhu, X.-Q. Chen, G. M. Stocks and Z. Y. Zhang, Phys. Rev. Lett. 105, 096404 (2010).

[29] D. J. Thouless, M. Kohmoto, M. P. Nightingale and M. den Nijs, Phys. Rev. Lett. 49, 405 (1982).

[30] Q. Niu and D. J. Thouless, J. Phys. A 17, 2453 (1984).

[31] D. N. Sheng, Z. Y. Weng, L. Sheng and F. D. M. Haldane, Phys. Rev. Lett. 97, 036808 (2006).

[32] E. Prodan, T. L. Hughes and B. A. Bernevig, Phys. Rev. Lett. 105, 115501 (2010).

[33] S. Murakami, S. Iso, Y. Avishai, M. Onoda and N. Nagaosa, Phys. Rev. B 76, 205304 (2007).

[34] S. Murakami, New J. Phys. 9, 256 (2007).

[35] Z.-F. Jiang, R.-L. Chu and S.-Q. Shen, Phys. Rev. B 81, $115322(2010)$.

[36] S. Datta, Electronic Transport in Mesoscopic Systems (Canmbridge University Press, Cambridge, U.K., 1995).

[37] A. MacKinnon, Z. Phys. B - Condensed Matter 59, 385 (1985).

[38] A. Weisse, G. Wellein, A. Alvermann and H. Fehske, Rev. Mod. Phys. 78, 275 (2006).

[39] E. Tang, J.-W. Mei and X.-G. Wen, Phys. Rev. Lett. 106, 236802 (2011).

[40] K. Sun, Z.-C. Gu, H. Katsura and S. Das Sarma, Phys. Rev. Lett. 106, 236803 (2011).

[41] T. Neupert. L. Santos, C. Chamon and C. Mudry, Phys. Rev. Lett. 106, 236804 (2011). 